\title{
algebraic proofs of Fermat's last theorem and Beals conjecture
}

\author{
James E. Joseph \\ Retired Professor, Department of Mathematics \\ Howard University \\ Washington, DC 20059 jjoseph@Howard.edu 35 E Street NW \#709 \\ where $c$ is odd, .Washington, DC 20001 j122437@yahoo.com
}

\section{ABSTRACT}

In this paper, the following statememt of Fermats Last Theorem is proved. If $x, y, z$ are positive integers $\pi$ is an odd prime and $z^{\pi}=x^{\pi}+y^{\pi}, x, y, z$ are all even. Also, in this paper, is proved (Beals conjecture) The equation $z^{\xi}=x^{\mu}+y^{v}$ has no solution in relatively prime positive integers $x, y, z$, with $\xi, \mu, v$ primes at least .

\section{$\S 1$. Fermats Last Theorem}

For other theorems named after Pierre de Fermat, see [1]. The 1670 edition of Diophantus' Arithmetica includes Fermat's commentary, particularly his "Last Theorem" (Observatio Domini Petri de Fermat). In number theory, Fermat's Last Theorem (sometimes called Fermat's conjecture, especially in older texts) states that no three positive integers $x, y$, and $z$ satisfy the equation $z^{\pi}=x^{\pi}+y^{\pi}$ for any integer value of $\pi$ greater than two. The case $\pi=2$ was known to have infinitely many solutions. This theorem was first conjectured by Pierre de Fermat in 1637 in the margin of a copy of Arithmetica where he claimed he had a proof that was too large to fit in the margin. The first proof agreed upon as successful was released in 1994 by Andrew Wiles, using cyclic groups, and formally published in 1995 [2], ]3], after 358 years of effort by mathematicians. The unsolved problem stimulated the development of algebraic number theory in the 19th century and the proof of the modularity theorem in the 20th century. It is among the most notable theorems in the history of mathematics It is known that if $x, y, z$ are relatively prime positive integers, $z^{4} \neq x^{4}+y^{4}[1]$. In view of this fact, it is only necessary to prove if $x, y, z$, are relatively prime positive integers, $\pi$ is an odd prime, $z^{\pi}=x^{\pi}+y^{\pi}$, then $x, y, z$, are each divisible by $\pi$. Before and since Wiles paper, many papers and books have been written trying to solve this problem in an elegant algebraic way, but none have suceeded. (See [1], and go to a search engine on the computer and search Fermats Last Theorem). In the remainder of this paper, $\pi$ will represent an odd prime. The special case $z^{4}=x^{4}+y^{4}$ is impossible. In view of this fact, it is only necessary to prove, if $x, y, z$, are positive integers, $\pi$ is an odd prime, $z^{\pi}=x^{\pi}+y^{\pi}$ Then $x, y, z$ are all even. Beals conjecture which states

The equation $z^{\xi}=x^{\mu}+y^{v}$ has no solution in relatively prime positive integers $x, y, z$, with $\xi, \mu, v$ primes at least 3 has not been given an algebraic solution. One million dollars has been offered for a solution.

Theorem. If $x, y, z$ are relatively prime positive integers and $z^{\pi}=x^{\pi}+y^{\pi}$, then $x, y, z$ are all even.

Proof. Since $z^{\pi}=x^{\pi}+y^{\pi}, x, y$ or $z$ is even. It will be shown that $x, y, z$ are all even.

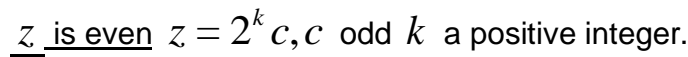

$$
\begin{gathered}
z^{\pi}=2^{k \pi} c^{\pi}=x^{\pi}+y^{\pi}=x^{\pi}\left(1+y^{\pi} x^{-\pi}\right)=y^{\pi}\left(1+x^{\pi} y^{-\pi}\right) \\
1+y^{\pi} x^{-\pi}=2^{k \pi}=1+x^{\pi} y^{-\pi}
\end{gathered}
$$

since

$$
(x, z)=(y, z)=1,
$$

so $x=y$.

$y$ is even $y=2^{k_{1}} c_{1}, c_{1}$ odd $k_{1}$ a positive integer.

$$
y^{\pi}=2^{k_{1}} \pi c_{1}^{\pi}=z^{\pi}-x^{\pi}=z^{\pi}\left(1-x^{\pi} z^{-\pi}\right)=z^{\pi}\left(1-x^{\pi} z^{-\pi}\right)
$$




$$
1-z^{\pi} x^{-\pi}=2^{k_{1} \pi}=1-x^{\pi} z^{-\pi}
$$

since

$$
(x, y)=(y, z)=1 \text {, }
$$

so $x=z$.

$\underline{x}$ is even $x=2^{k_{2}} c_{2}, c_{2}$ odd $k_{2}$ a positive integer.

$$
\begin{gathered}
x^{\pi}=2^{k_{2} \pi} c_{2}^{\pi}=z^{\pi}-y^{\pi}=z^{\pi}\left(1-y^{\pi} z^{-\pi}\right)=z^{\pi}\left(1-y^{\pi} z^{-\pi}\right) ; \\
1-z^{\pi} y^{-\pi}=2^{k_{2}} \pi=1-y^{\pi} z^{-\pi},
\end{gathered}
$$

since

$$
(x, y)=(x, z)=1,
$$

so $y=z$.

Fermat's Last Theorem If $x, y, z$ are relatively prime positive integers, and $\pi$ is an odd prime, then $z^{\pi} \neq x^{\pi}+y^{\pi}$.

Proof. If $z^{\pi}=x^{\pi}+y^{\pi}$, then $z, y, x$ are all even .

The same technique may be used to establish the following result.

\section{$\S 2$. Beals conjecture}

The equation $z^{\xi}=x^{\mu}+y^{v}$ has no solution in relatively prime positive integers $x, y, z$, with $\xi, \mu, v$ primes at least 3 .

Proof. Again, $x, y$ or $z$ must be even.

$\underline{z}$ is even $z=2^{k} c, c$ odd $k$ a positive integer.

$$
\begin{gathered}
z^{\xi}=2^{k \xi} c^{\xi}=x^{\mu}+y^{v}=x^{\mu}\left(1+y^{v} x^{-\mu}\right)=y^{v}\left(1+x^{m} u y^{-v}\right) \\
1+y^{v} x^{-\mu}=2^{k \xi}=1+x^{\mu} y^{-v}
\end{gathered}
$$

since

$$
(x, z)=(y, z)=1 \text {, }
$$

so $\left(x^{\mu}\right)^{2}=\left(y^{v}\right)^{2}, x^{\mu}=y^{v}$.

$y$ is even $y=2^{k_{1}} c_{1}, c_{1}$ odd $k_{1}$ a positive integer.

$$
\begin{aligned}
y^{v}=2^{k_{1} v} c_{1}^{v}=z^{\xi}-x^{\mu} & =z^{\xi}\left(1-x^{\mu} z^{-\xi}\right)=z^{\xi}\left(1-x^{\mu} z^{-\xi}\right) ; \\
1+z^{\xi} x^{-m u} & \left.=2^{k_{1}} x i\right)=1+x^{\mu} z^{-\xi},
\end{aligned}
$$

since

$$
(x, y)=(y, z)=1 \text {, }
$$

so $x^{\mu}=z^{\xi}$.

$\underline{x}$ is even $x=2^{k_{2}} c_{2}, c_{2}$ odd $k_{2}$ a positive integer.

$$
x^{\mu}=2^{k_{2} \mu} c_{2}^{\xi}=z^{\xi}-y^{v}=z^{\xi}\left(1-y^{v} z^{-\xi}\right)=z^{\xi}\left(1-y^{v} z^{-\xi}\right) ;
$$




$$
1+z^{\xi} y^{-n u}=2^{k_{2} \pi_{1}}=1+y^{v} z^{-\xi}
$$

since

$$
(x, y)=(x, z)=1, y^{v}=z^{\xi} .
$$

In closing, an alternative proof is given for Beals conjecture.

\section{$\S 3$. An alternative proof to Beals conjecture}

Any solution $x, y, z$ to the equation $z^{\xi}=x^{\mu}+y^{v}$ with $\xi, \mu, v$ primes at least 3 must all be divisible bv 2 .

Proof.

$$
\left(z^{\xi}\right)^{\xi}=\left(x^{\xi}\right)^{\mu}+\left(y^{\xi}\right)^{v}=\left(x^{\mu}\right)^{\xi}+\left(y^{v}\right)^{\xi},
$$

and by Fermats Last Theorem., $z^{\xi}, x^{\mu}, y^{\nu}$ and $x, y, z$ are all divisible bv 2 .

\section{REFERENCES}

[1] H. Edwards, Fermat's Last Theorem:A Genetic Introduction to Algebraic Number Theory, Springer-Verlag, New York, (1977).

[2] A. Wiles, Modular ellipic eurves and Fermat's Last Theorem, Ann. Math. 141 (1995), 443-551.

[3] A. Wiles and R. Taylor, Ring-theoretic properties of certain Heche algebras, Ann. Math. 141 (1995), 553-573. 